\title{
Composición de carteras de inversión en títulos de rente fija utilizando modelos de optimización robusta por escenarios
}

\author{
Composition of portfolios in fixed income securities using robust \\ optimization models for scenarios
}

\author{
Rafael Cruz', Pablo Cortés ${ }^{2}$ \\ I Endesa. Av. De la Borbolla, 5, 4l 005 Sevilla. \\ 2 Ingeniería de Organización. Escuela Técnica Superior de Ingenieros. Universidad de Sevilla. \\ Camino de los Descubrimientos, s/n, 41092 Sevilla \\ pca@esi.us.es
}

Fecha de recepción: 08-02-2010

Fecha de aceptación: 18-06-2010

Resumen: En el presente trabajo se estudian y analizan los Modelos de Optimización Robusta como herramientas eficientes para la Gestión del Riesgo de Títulos de Renta Fija. La parte teórica del trabajo se complementa con el estudio del Modelo de Optimización por Escenarios (aplicado al problema de selección de cartera y partiendo de una dotación inicial indeterminada), poniéndose de manifiesto la eficiencia de los resultados obtenidos por su aplicación. Se presenta un problema consistente en la composición de carteras de inversión de renta fija el cual se resuelve mediante la utilización de Modelos de Optimización Robusta por escenarios de los tipos MM y MAD. Por último, se acomete un análisis de sensibilidad ante las distintas posibilidades de ocurrencia de los diferentes escenarios para cada uno de los modelos.

Palabras clave: Cartera de inversión, Optimización robusta, Escenario, Rendimiento esperado, Error coordinado.

\begin{abstract}
In this paper we study and analyze the Robust optimization models as efficient tools for risk Management Fixed Income Securities. The theoretical part of work is complemented by the study by Scenario Optimization model (applied to the problem of portfolio selection and starting from an initial endowment indeterminate), getting to show the efficiency of the results obtained on application. A problem is presented consisting in managing portfolio of fixed income investment which is solved using Robust Optimization Models for scenarios of the types MM and MAD.Finally, it undertakes a sensitivity analysis of the different possibilities of occurrence of different scenarios for each of the models.
\end{abstract}

Key words: Portfolio investment, robust optimization, Stage, Expected Return, Error coordinated.

\section{Introducción}

El estudio sectorial de inversiones en títulos de renta fija goza de una enorme tradición en el mercado de inversiones, existiendo numerosos fondos de inversión que invierten exclusivamente en renta fija. Son los llamados «fondos de renta fija» disponibles en el catálogo de cualquier banco, caja o sociedad de valores. El principal motivo de su selección es, además de ofrecer una mayor liquidez, la seguridad de la inversión, es decir, ofrecer una mayor estabilidad de la misma.

El problema de selección de cartera es muy complejo ya que existen múltiples fuentes de incertidumbre y múltiples criterios de selección. El binomio rentabi- lidad-riesgo es el principio básico en el planteamiento de los modelos con el consiguiente conflicto de objetivos, ya que para lograr un mayor rendimiento se deben adoptar estrategias más arriesgadas.

La principal referencia histórica en la materia se debe al Modelo de Markowitz (1952). El objetivo del autor fue plantear un modelo de conducta racional del decisor para la selección de carteras de títulos-valores con liquidez inmediata (en su modelo, la liquidez del título es inmediata al final del periodo de referencia). Propuso un modelo de media-varianza (MV) de selección de cartera. En este modelo la rentabilidad esperada y el riesgo de la inversión se miden por la media y la varianza de las rentabilidades históricas. 
Posteriormente, algunos autores propusieron diversos modelos que introducían distintas modificaciones y mejoras al Modelo de Markowitz. Sharpe (1964), planteaba una simplificación consistente en suponer la existencia de una relación lineal entre el rendimiento del título y el de la cartera de mercado. Ello significaba que se podía definir el riesgo de la cartera sin utilizar las covarianzas, suponiendo una gran simplificación en el cálculo. Michaud (1989), realizó una aproximación al tratamiento de la inestabilidad de las soluciones ante cambios en las rentabilidades esperadas. Parte del principio de que la frontera eficiente no es una simple línea sino que es una banda que representa un intervalo de confianza. Cambios en los parámetros, pueden conducir a carteras dentro de ese mismo intervalo de confianza o fuera del mismo. Cuando estas nuevas carteras derivadas de nuevos parámetros lleven a soluciones dentro del mismo intervalo de confianza, la cartera inicialmente encontrada no debe ser modificada. Solo debe serlo cuando la nueva solución quede fuera del intervalo de confianza hallado previamente.Y, por último, Chopra y Ziemba (1993), los cuales estudiaron las consecuencias de los errores de estimación en la rentabilidad esperada y en las varianzas y covarianzas en el modelo de Markowitz. Llegaron a la conclusión de que los errores en la rentabilidad esperada tienen una importancia del orden de I I veces mayor que los errores en la estimación de la varianza y 20 veces mayor que los de la covarianza a los efectos de la desviación sobre el verdadero óptimo. Además, para los inversores más adversos al riesgo, los errores en la rentabilidad esperada son mucho más importantes que para aquellos con menor aversión al riesgo. Esto es, la solución obtenida está más alejada del verdadero óptimo.

Roy (1952), Kataoca (1963) o Telser (1955) han realizado estudios sobre el Modelo Safety First, el cual antepone la seguridad del inversor ante cualquier posible incremento del retorno. Alternativas a los modelos citados son los modelos que requieren medidas a partir de datos históricos y la experiencia de gestores expertos (Tanaka y Guo, 1999), así como la consideración de escenarios y su aplicación en optimización robusta, con bases en la programación por objetivos y la consideración de restricciones débiles (Mulvey, Vanderbei y Zenios, 1995). Aunque tratar distintos escenarios ha sido tradicional en la gestión científico-empresarial, y la idea ya estaba presente en los estados de la naturaleza de la Teoría de la Decisión de Von Neumann y Morgenster, en selección de cartera no ha tenido su impulso hasta el Modelo de Escenarios y Factores de Markowitz y Perold (1981).
El uso de la optimización robusta en la toma de decisiones permite considerar directamente la aversión al riesgo (Bai, Carpenter y Mulvey, 1997), siendo esta una de sus ventajas frente a la programación estocástica. Dembo (1992), Escudero (1995), Golub ( 1994) y Vassiadou-Zeniou y Zenios (1996) han aplicado sus modelos a la planificación y gestión financiera de modelos de optimización robusta. Es en este aspecto donde se centra este trabajo.

La optimización robusta consiste en una integración de la programación por objetivos basados en los escenarios posibles. Con su aplicación se generan una serie de soluciones que progresivamente se van convirtiendo en menos sensibles a las posibles realizaciones de los diferentes escenarios. La solución óptima del problema es robusta respecto a la optimalidad si se mantiene «cerrada» en el óptimo. En el caso de que se mantenga admisible para cualquier realización, se dice que el modelo es robusto.

La aplicación de los Modelos de Optimización Robusta desarrollados aporta una gran solidez a la toma de decisiones en las empresas con inversiones en títulos de renta fija

La variedad de los escenarios y las volatilidades de los diferentes mercados en los que un gestor puede actuar hacen imprescindible un estudio a fondo del riesgo de la inversión. La finalidad de dicho estudio es que el gestor pueda disponer de herramientas que le faciliten su tratamiento, asegurando y rentabilizando al máximo sus inversiones y mejorando, como consecuencia, la competitividad de la empresa. La gestión sistemática de los riesgos asegura el éxito de las inversiones y la previsibilidad de los indicadores de las mismas.

Entre las principales aplicaciones actuales de Optimización Robusta se encuentra la aplicación al problema del Cash-Flow matching con incertidumbre (Canós, M. J. y Ventura, M. 1999) en el que se pretende determinar el importe de la inversión a realizar en el momento inicial, con el objeto de atender a una posible serie de pagos a lo largo de un determinado periodo de planificación. La gestión del Riesgo de Interés (Dembo, I991), en el que se describen los parámetros aleatorios del problema (tipos de interés) a través de escenarios con sus correspondientes probabilidades, permitiendo introducir la subjetividad del decisor al elegir los escenarios y asignar probabilidades. Y también al Modelo de Recurso Total (Rockafellar y Wets, 1993 y Escudero, L. 1995) en el que todas las decisiones son ajustadas en el tiem- 
po cuando se consigue una nueva información sobre las mismas, excepto las correspondientes al primer periodo de tiempo, tratando que las decisiones sean consistentes con la información disponible en cada periodo de tiempo dado.

En el presente trabajo se aplican Modelos de Optimización Robusta para realizar un estudio sobre la Gestión del Riesgo en las carteras de inversión con títulos de renta fija. Para ello, se identifica, analiza y evalúa cuantitativamente el mismo.

Además, resuelve un modelo de optimización por escenarios aplicado al problema de selección de cartera con dotación inicial indeterminada.

El modelo propone el capital a invertir y una cartera diferente para cada uno de los escenarios. Como puede que sea improbable que una solución pueda cumplir simultáneamente los criterios de robustez para la admisibilidad y para la optimalidad, se construye un modelo coordinado que combina ambos conceptos de forma adecuada.

En el apartado segundo se muestran los modelos de programación matemática que se desarrollan para la toma de decisiones basados en modelos de optimización robusta por escenarios, en los que se busca minimizar el error coordinado.

El apartado tercero recoge un análisis de los resultados obtenidos, así como un análisis de sensibilidad para cada uno de los distintos modelos.

Por último, en el cuarto apartado se expone una comparación de los resultados de los modelos; para terminar con las principales conclusiones en la sección quinta.

\section{Modelos de programación matemática para la toma de decisiones de cartera de inversión}

\section{I. Modelado del problema}

El estudio parte de la determinación de un conjunto de escenarios, $s=1, \ldots, S, y$ unos rendimientos esperados de cada bono, $i=1, \ldots$, , conocido para cada escenario (que se notará por $r_{i s}$ ). A partir de aquí se puede obtener la frontera eficiente de cada escenario resolviendo el siguiente modelo basado en el de Markowitz que determina la cartera eficiente para cada uno de los escenarios de forma independiente, y que se ha denominado Modelo COI (cartera óptima independiente):

$$
\sigma^{2}=\sum_{i=1}^{n} \sum_{j=1}^{n} \sigma_{i j} x_{i s} x_{j s}
$$

Min

$$
\sum_{i=1}^{n} r_{i s} x_{i s}=r_{s} C_{s}
$$

s.a.

$$
\begin{aligned}
& \sum_{i=1}^{n} x_{i s}=C_{s} \\
& x_{i s} \geq 0 \quad \forall i=1, \ldots, n
\end{aligned}
$$

Donde $\sigma^{2}$, determina la varianza de la cartera; $x_{i s}$ es la cantidad invertida en el activo en el escenario $s, r_{\text {is }}$ es la rentabilidad esperada del activo $i$ en el escenario, que se considera dato tal y como se ha señalado, $C_{s}$ es el capital total invertido en la cartera en el escenario $s$ y $r_{s}$ es la rentabilidad esperada de la cartera en el escenario s. Ambos son datos del modelo.

La resolución del modelo para cada escenario variando el valor del parámetro $r_{s}$ permite obtener la frontera eficiente de ese escenario.

La consideración del hecho de que el escenario que se vaya a producir de forma fehaciente al final del proceso es algo desconocido, pero que, en cambio, sí puede estimarse una cierta probabilidad asociada al hecho de que tenga lugar un escenario u otro, conduce a la consideración de un modelo coordinado que combina los conceptos de admisibilidad y optimalidad adecuadamente. Es decir, un modelo robusto que controle suficientemente el riesgo que el inversor asume al realizar la inversión y se mantenga lo más cerca posible de la solución óptima, sea cual sea el escenario que se produzca. El concepto de robustez que aquí se introduce trata de paliar los problemas que presenta el Modelo de Markowitz. El primer problema es una excesiva exposición a variaciones de rentabilidades futuras, mediante la consideración de distintos escenarios. Los valores de las varianzas con respecto a las rentabilidades esperadas en intervalos cortos de tiempo (por ejemplo, un año) son muy grandes. Es decir, el error de predicción en esos periodos es también elevado. Por otra parte, es extremadamente sensible a los valores de las rentabilidades esperadas, de tal forma que unas pequeñas variaciones de las mismas suponen carteras con estructuras muy diferentes en su composición. 
El objetivo del modelo es minimizar el error coordinado de la inversión en el activo respecto al escenario s. Dicho error será la desviación relativa en el beneficio esperado respecto al del óptimo de cada escenario. El modelo se basa en la propuesta de Canós y Ventura (1999). Sin embargo, este modelo precisa de ciertas modificaciones en nuestra opinión para alcanzar un mayor grado de satisfacción en sus resultados y que a continuación se describen.

En primer lugar, dicho modelo define el error coordinado como $\varepsilon^{s}(x)=r_{s} C_{s}-\sum_{i=1}^{n} r_{i} x_{i}$, pero esta definición trae como consecuencia que la composición de la cartera solución del modelo coordinado es idéntica a la óptima del escenario más favorable donde el capital a invertir es el máximo considerado, al igual que el riesgo. El error coordinado respecto a esta cartera es cero y negativo para las carteras óptimas del resto de escenarios siendo, por tanto, ésta la cartera solución del modelo coordinado. Por ello, creemos conveniente definir el error coordinado de $x$ respecto al escenario s como la desviación relativa en el beneficio esperado de la solución $x$ respecto al del óptimo de cada escenario, expresada en términos porcentuales en lugar de en unidades monetarias. Es decir:

$\varepsilon_{s}(x)=\frac{r_{s} C_{s}-\sum_{i=1}^{n} r_{i s} x_{i}}{r_{s} C_{s}}$.

Además, se ha considerado una rentabilidad esperada distinta para cada título en función del escenario previsto, ya que dicho modelo no considera que las rentabilidades esperadas de cada título deban ser distintas en función del escenario que se considere.

En segundo lugar, también se ha eliminado la restricción $y_{s}+\sum_{i=0 ; i \neq s-1 ; i \neq s+1}^{s} y_{i} \leq 1$ ya que ésta impide que el capital a invertir sea igual a uno de los capitales establecidos en la partición.

Así, se proponen dos modelos coordinados. En el primero se minimiza el máximo error producido en el escenario, s, más desfavorable, y se ha denominado Modelo MM (min-max error).

Min $\varepsilon$

Sujeto a:

$P_{s} \varepsilon_{s}(x) \leq \varepsilon, s=1, \ldots, S$

$$
\begin{aligned}
& C_{\min }\left(1-\frac{C_{\min }-C_{s}}{C_{\min }} y^{s}\right) \leq C(x) \quad s=1, \ldots, s-1 \\
& C_{\max }\left(1-\frac{C_{\max }-C_{s+1}}{C_{\max }} y_{s}\right) \geq C(x) \quad s=1, \ldots, s-1 \\
& L(x) \leq L\left(x_{s+1}\right) y_{s}+L\left(x_{s}\right)\left(1-y_{s}\right), \quad s=1, \ldots, s-1 \\
& I \leq \sum_{s=1}^{s-1} y_{s} \leq 2 \\
& \varepsilon, x \geq 0, y_{s} \in\{0,1\}
\end{aligned}
$$

Para la construcción de estos modelos el riesgo de la cartera se medirá a través de la varianza de los rendimientos históricos de la misma, siendo $\sigma_{i j}$ la covarianza del rendimiento del activo $i$ con el del rendimiento del activo $j$.

Como variables del modelo se toman: el error coordinado $(\varepsilon)$; la cantidad invertida en el activo $i\left(x_{i}\right)$ que ahora corresponde a la inversión que finalmente se acomete $y$ es independiente del escenario que se pueda o no producir apriorísticamente; el capital total coordinado asociado $[C(x)]$ que queda, por tanto, indeterminado; el riesgo de inversión del vector de activos $x=\left(x_{1}, \ldots, x_{i}, \ldots, x_{n}\right)[L(x)]$ y la variable binaria que establece el escenario observado $s\left(y_{s}\right)$.

Como datos a tener en cuenta se tomará la probabilidad de ocurrencia del escenario $s\left(P_{s}\right)$, el capital mínimo a invertir $\left(C_{\min }\right)$ y el capital máximo a inver$\operatorname{tir}\left(C_{\max }\right)$.

Por último, el conjunto de distintos rating de bonos $i \in I: i=1,2, \ldots, n(I)$ y el conjunto de escenarios (S) serán los parámetros a incluir.

Una vez resuelto el modelo, los valores de las variables $x_{i}$ y $C(x)$ serán los de la composición de la cartera real $y$ del capital que se va a invertir en ella, respectivamente.

El segundo modelo que se presenta coincide con el anterior y sólo se diferencia del anterior en la función objetivo en la cual se busca minimizar el error medio ponderado por la probabilidad de ocurrencia del escenario s. Se ha denominado Modelo MAD (minimum absolute deviation), siendo la función objetivo referida Min $\varepsilon=\sum_{s=1}^{S} P_{s}\left|\varepsilon_{s}\right|$. 


\subsection{Construcción de datos para el estudio}

En primer lugar, se recogen los datos relativos a cada bono, seguidamente se realiza un cálculo del riesgo de la cartera, se construyen los escenarios y, por último, se calculan los rendimientos esperados en cada escenario.

Los bonos que se manejarán para la experimentación en este trabajo son los bonos corporativos estadounidenses. Los bonos son emitidos generalmente en múltiplos de $\$ 1.000$ (también conocido como valor nominal o valor par del bono). Se manejan, como posibilidades de inversión, un grupo de 24 bonos pertenecientes al mercado secundario estadounidense de bonos corporativos con vencimientos comprendidos entre 3 y 10 años. Concretamente ocho bonos con vencimiento a 3 años (20|2), ocho con vencimiento a 5 años (20 I 4) y ocho con vencimiento a I 0 años (20 I9). Para todos los casos se parte de 2009 como año inicial.

A partir de los datos de todas las transacciones realizadas sobre cada bono durante el año 2008 (obtenidos de FINRA en www.finra.org), se construye para cada bono una tabla donde se muestran los precios históricos del año 2008 para el bono WFC.JL (Tablas I y 2).

En esta tabla puede consultarse, entre otros datos, la fecha (en formato $\mathrm{mm} / \mathrm{dd} / \mathrm{aaaa}$ ), la hora de cada transacción, el tamaño de la misma, el precio del bono en cuestión para esa transacción y el rendimiento al vencimiento para ese precio.

Los precios de negociación de cada uno de los 24 bonos para todas las transacciones realizadas durante el año 2008, así como el resto de la información general de cada bono, son los datos utilizados para la construcción de los escenarios, el cálculo de los rendimientos esperados de cada bono y el cálculo de la matriz de covarianzas, necesaria para evaluar el riesgo.

Con respecto a los criterios de elección de bonos, la elección de los 24 bonos se ha realizado siguiendo los siguientes criterios:

- Bonos muy activos: El problema tratado es el de selección de cartera con horizonte de inversión de un año, transcurrido el cual, se venderían los títulos, procediendo a consumir o a reinvertir el dinero recibido. Para no tener que considerar en el modelo el riesgo de liquidez, o riesgo de que las posibilidades de vender el bono sean limitadas, se han seleccionado bonos con un alto número y volumen de transacciones durante el año
2008 con los que presumiblemente disminuiría la exposición a este riesgo.

Tabla 1

Transacciones correspondientes al mes de enero para el bonoWFC.JL

\begin{tabular}{|c|c|c|c|c|}
\hline Date & Time & Quantity & Price & Yield \\
\hline $01 / 02 / 2008$ & $9: 41: 50$ & 46.000 & 104.764 & 4.876 \\
\hline $01 / 02 / 2008$ & $9: 41: 55$ & 46.000 & 105.786 & 4.617 \\
\hline $01 / 02 / 2008$ & $9: 43: 00$ & 4.000 & 104.686 & 4.895 \\
\hline $01 / 02 / 2008$ & $9: 43: 41$ & 4.000 & 105.686 & 4.642 \\
\hline $01 / 03 / 2008$ & $10: 55: 34$ & 50.000 & 105.245 & 4.753 \\
\hline $01 / 03 / 2008$ & $10: 55: 35$ & 50.000 & 106.015 & 4.559 \\
\hline $01 / 04 / 2008$ & $12: 07: 14$ & $5 M M+$ & 105.500 & 4.687 \\
\hline $01 / 04 / 2008$ & $12: 13: 00$ & $5 M M+$ & 105.532 & 4.679 \\
\hline $01 / 15 / 2008$ & |3:09:14 & | 40.000 & 104.467 & 4.944 \\
\hline $01 / 15 / 2008$ & 13:09:17 & 140.000 & 104.850 & 4.846 \\
\hline $01 / 16 / 2008$ & $10: 48: 00$ & 5.000 & 106.568 & 4.407 \\
\hline $01 / 16 / 2008$ & 13:03:00 & 20.000 & 106.361 & 4.459 \\
\hline $01 / 16 / 2008$ & | 3:34:00 & 10.000 & 106.355 & 4.461 \\
\hline $01 / 16 / 2008$ & 13:34:19 & 10.000 & 107.205 & 4.248 \\
\hline $01 / / 8 / 2008$ & $10: 22: 52$ & 15.000 & 105.500 & 4.675 \\
\hline $01 / 23 / 2008$ & 10:08:00 & 10.000 & 107.653 & 4.129 \\
\hline $01 / 24 / 2008$ & $12: 22: 00$ & 3.000 & 106.924 & 4.311 \\
\hline $01 / 24 / 2008$ & | 6:56:00 & 3.000 & 106.224 & 4.487 \\
\hline $01 / 24 / 2008$ & |6:56: I | & 3.000 & 106.490 & 4.419 \\
\hline $01 / 24 / 2008$ & | 6:56: | | & 3.000 & 106.224 & 4.487 \\
\hline $01 / 25 / 2008$ & 13:16:22 & 25.000 & 105.000 & 4.799 \\
\hline $01 / 28 / 2008$ & $16: 19: 36$ & 14.000 & 107.250 & 4.227 \\
\hline $01 / 28 / 2008$ & $16: 19: 36$ & 14.000 & 106.200 & 4.491 \\
\hline $01 / 28 / 2008$ & $16: 19: 36$ & 14.000 & 107.250 & 4.227 \\
\hline $01 / 28 / 2008$ & $16: 19: 36$ & 14.000 & 108.050 & 4.227 \\
\hline $01 / 28 / 2008$ & $16: 19: 36$ & 14.000 & 108.050 & 4.227 \\
\hline $01 / 28 / 2008$ & $16: 19: 36$ & 14.000 & 107.250 & 4.227 \\
\hline $01 / 28 / 2008$ & $16: 19: 39$ & 14.000 & 106.170 & 4.499 \\
\hline $01 / 30 / 2008$ & | 4:00:00 & 30.000 & | 05.75 | & 4.603 \\
\hline $01 / 30 / 2008$ & $14: 12: 00$ & 89.000 & 106.057 & 4.525 \\
\hline $01 / 30 / 2008$ & $14: 12: 33$ & 89.000 & 106.087 & 4.517 \\
\hline $01 / 30 / 2008$ & $14: 12: 33$ & 89.000 & 106.812 & 4.334 \\
\hline $01 / 31 / 2008$ & $\mid 2: 51: 00$ & 2.000 .000 & 106.925 & 4.304 \\
\hline $01 / 31 / 2008$ & $|2: 5|: 00$ & 2.000 .000 & 106.925 & 4.304 \\
\hline $01 / 31 / 2008$ & $12: 52: 00$ & 2.000 .000 & 107.139 & 4.250 \\
\hline
\end{tabular}

Tabla 2

Precios Históricos

\begin{tabular}{|c|c|}
\hline Mes & Precio (P) \\
\hline ene-08 & 106.615 \\
\hline feb-08 & 107.077 \\
\hline mar-08 & 104.958 \\
\hline abr-08 & 104.606 \\
\hline may-08 & 101.116 \\
\hline jun-08 & 103.329 \\
\hline jul-08 & 102.438 \\
\hline ago-08 & 101.417 \\
\hline sep-08 & 99.123 \\
\hline oct-08 & 100.522 \\
\hline nov-08 & 102.474 \\
\hline dic-08 & 102.471 \\
\hline
\end{tabular}


- Bonos no amortizables anticipadamente:Tampoco se ha tenido en cuenta el riesgo de amortización anticipada en los modelos. Para ello, los bonos empleados en este estudio son bonos que no incorporan esta opción para el inversor (Noncallable bonds).

- Bonos de calificaciones similares. Es decir, aunque es posible tener en cuenta el riesgo de insolvencia en el análisis del rendimiento esperado de los bonos, en este caso no se ha considerado, seleccionándose, como posibilidades de inversión, bonos de calificación similares para que fueran comparables en términos de riesgo de insolvencia. Todos los bonos considerados pertenecen al grupo definido como inversión «por encima de la media» o «superior al grado medio» calificados con ratings entre $A-y$ A + por Standard \& Poor's y Fitch y entre A3 y AI por Moody's.

Luego las hipótesis de los bonos considerados en los modelos son el bajo riesgo de liquidez, el no ser amortizables anticipadamente y el tener calificaciones similares.

Se ha considerado, como precio de mercado del bono en cada mes $(P)$, la media del precio de negociación de todas las transacciones realizadas durante ese mes. Los precios se expresan normalmente en porcentaje respecto al nominal ( 1.000 \$ para este tipo).

A partir de esta tabla se construye la de rendimientos históricos (Tabla 3) utilizando la definición de rendimiento actual ( $r a=\frac{Q}{P} \times 100$, donde $Q$ es la tasa de interés del cupón):

Tabla 3

Rendimientos históricos

\begin{tabular}{|c|c|}
\hline Mes & Rendimiento (ra) \\
\hline ene-08 & 5,76 \\
\hline feb-08 & 5,76 \\
\hline mar-08 & 5,72 \\
\hline abr-08 & 5,82 \\
\hline may-08 & 5,89 \\
\hline jun-08 & 5,95 \\
\hline jul-08 & 5,97 \\
\hline ago-08 & 5,99 \\
\hline sep-08 & 6,08 \\
\hline oct-08 & 6,13 \\
\hline nov-08 & 6,08 \\
\hline dic-08 & 5,98 \\
\hline
\end{tabular}

Repitiendo la operación, se obtiene para cada bono un vector de 12 elementos con los rendimientos actuales de cada mes del año 2008. A partir de estos vectores se obtiene la matriz de covarianzas $(24 \times 24)$, donde la covarianza de dos bonos i y j es igual a $\sigma_{i j}=\frac{1}{12} \sum_{t=1}^{12}\left(R_{i t}-\overline{R_{i}}\right)\left(R_{j t}-\overline{R_{j}}\right)$.

Para los rendimientos históricos se ha utilizado el rendimiento actual en lugar del rendimiento al vencimiento debido a que este último es la tasa interna de rentabilidad (TIR) de la inversión, y, dado que se han considerado bonos con distinto horizonte de vencimiento, no sería correcto utilizar esta medida al construir la matriz de covarianzas. Así, para obtener los rendimientos esperados se ha calculado, en primer lugar, la variación del rendimiento al vencimiento, seguidamente el precio de mercado al final del periodo de inversión y, por último, el rendimiento esperado.

La construcción de escenarios se ha realizado en base a la estructura de los tipos de interés existente a fecha 01/01/2009 para los bonos corporativos de calificación A. A través de la página de FINRA se han obtenido los datos de rendimiento al vencimiento para los distintos plazos de vencimiento del conjunto de bonos corporativos con esta calificación (Tabla 4).

Se han supuesto 5 posibles escenarios futuros, que representarían la ETTI al final del periodo de inversión $(01 / 01 / 10)$. En cuanto a los movimientos de la curva se ha supuesto que se producen desplazamientos paralelos de la curva de rendimientos. De estos 5 escenarios supuestos, el escenario central s3 sería el caso de un mantenimiento de tipos de interés, para el que la curva de rendimientos sería la misma que al comienzo del periodo de inversión. Además, de este escenario central, existirían dos escenarios favorables s4 y s5 con bajadas de tipos tales que supongan una disminución en el rendimiento al vencimiento (TIR) del conjunto de bonos considerados de 100 y 200 puntos básicos respectivamente (escala mínima de variación porcentual utilizada al comparar rendimientos de títulos de renta fija, representando la centésima parte de un punto porcentual. 100 puntos básicos es igual a 1\%.) y otros dos escenarios desfavorables s2 y s I con subidas de tipos tales que supongan una aumento del rendimiento al vencimiento de 100 y 200 puntos básicos.

Las curvas de rendimientos que representan los 5 escenarios se muestran en la Figura I. 
Figura I

Curvas de rentabilidades de los distintos escenarios

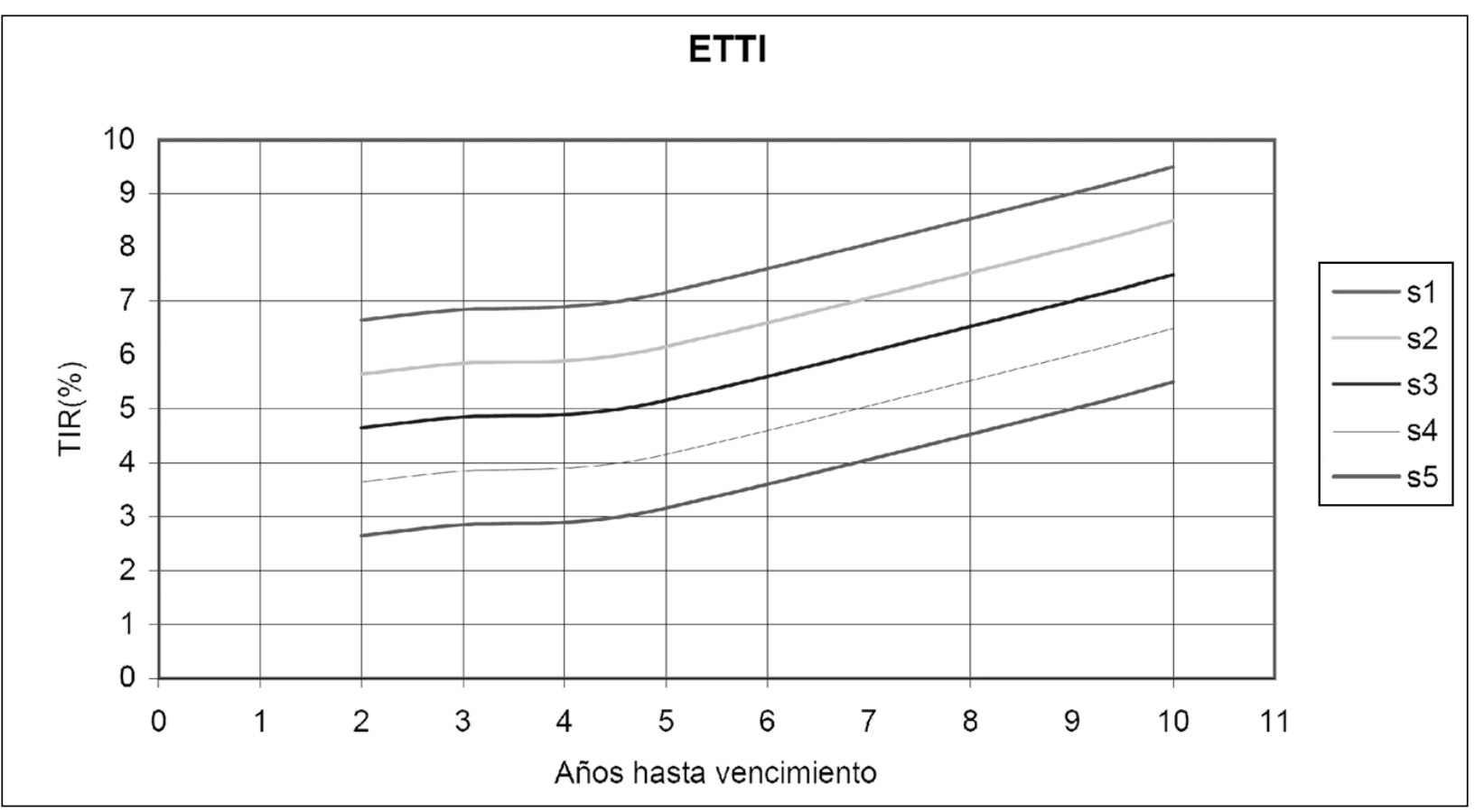

Tabla 4

Variaciones porcentuales del rendimiento al vencimiento (TIR)

\begin{tabular}{|c|c|c|c|c|c|c|}
\cline { 3 - 7 } \multicolumn{1}{c|}{} & bono & \multicolumn{5}{c|}{ bono 2 años } \\
\cline { 3 - 7 } \multicolumn{1}{c|}{3 años } & s5 & s4 & s3 & s2 & sl \\
\hline \multirow{3}{*}{ TIR } & 4,85 & 2,65 & 3,65 & 4,65 & 5,65 & 6,65 \\
\hline & \multicolumn{6}{|c|}{ Varlación \% } \\
\cline { 2 - 7 } & $-45 \%$ & $-25 \%$ & $-4 \%$ & $16 \%$ & $37 \%$ \\
\hline
\end{tabular}

\begin{tabular}{|c|c|c|c|c|c|c|}
\cline { 3 - 7 } \multicolumn{1}{c|}{} & bono & \multicolumn{5}{c|}{ bono 4 años } \\
\cline { 3 - 7 } \multicolumn{1}{c|}{5 años } & s5 & s4 & s3 & s2 & sl \\
\hline \multirow{2}{*}{ TIR } & 5,16 & 2,90 & 3,90 & 4,90 & 5,90 & 6,90 \\
\hline & & $-44 \%$ & $-24 \%$ & $-5 \%$ & $14 \%$ & $34 \%$ \\
\hline
\end{tabular}

\begin{tabular}{|c|c|c|c|c|c|c|}
\cline { 3 - 7 } \multicolumn{1}{c|}{} & bono & \multicolumn{5}{c|}{ bono 9 años } \\
\cline { 3 - 7 } \multicolumn{1}{c|}{} & 10 años & $s 5$ & $s 4$ & $s 3$ & $s 2$ & $s$ | \\
\hline \multirow{2}{*}{ TIR } & 7,50 & 5,00 & 6,00 & 7,00 & 8,00 & 9,00 \\
\hline \multirow{3}{*}{} & $-33 \%$ & $-20 \%$ & $-7 \%$ & $7 \%$ & $20 \%$ \\
\cline { 3 - 7 } & & \multicolumn{5}{|c|}{ Variación \% } \\
\cline { 2 - 7 }
\end{tabular}

La curva central representa tanto la ETTI al comienzo del periodo de inversión como la del escenario s3 en el que los tipos no han variado al final de periodo.

A partir de las curvas de rendimientos (figura) se calcula la variación porcentual que se produce en el rendimiento al vencimiento (TIR) del mercado de bonos con calificación A desde el instante inicial de la inversión al instante final, según se dé un escenario u otro.
Los argumentos necesarios para estas dos funciones se organizan como se indica en la Tabla 5.

A continuación, se calcula el precio de mercado que tendría un bono si en el momento final de la inversión tiene que proporcionar un rendimiento un 20\% más alto que el que tenía en el instante inicial para competir en el mercado de bonos.

En la Tabla 6 se sustituye la fecha de adquisición por la fecha de venta $(01 / 01 / 2010)$ y el rendimiento anual 
deseado, $7,07 \%$, por $7,07 ¥(1+0,20)=8,49 \%$, es decir, un $20 \%$ más del que proporcionaba en el instante inicial de la inversión.
Partiendo de la Tabla 5 y añadiendo la fecha de venta $(01 / 01 / 2010)$ y el precio de venta estimado para este escenario $(944,5$ I \$) se construye la Tabla 7.

Tabla 5

Cálculo del precio y rendimiento del bono en el instante inicial de la inversión mediante la herramienta Excel

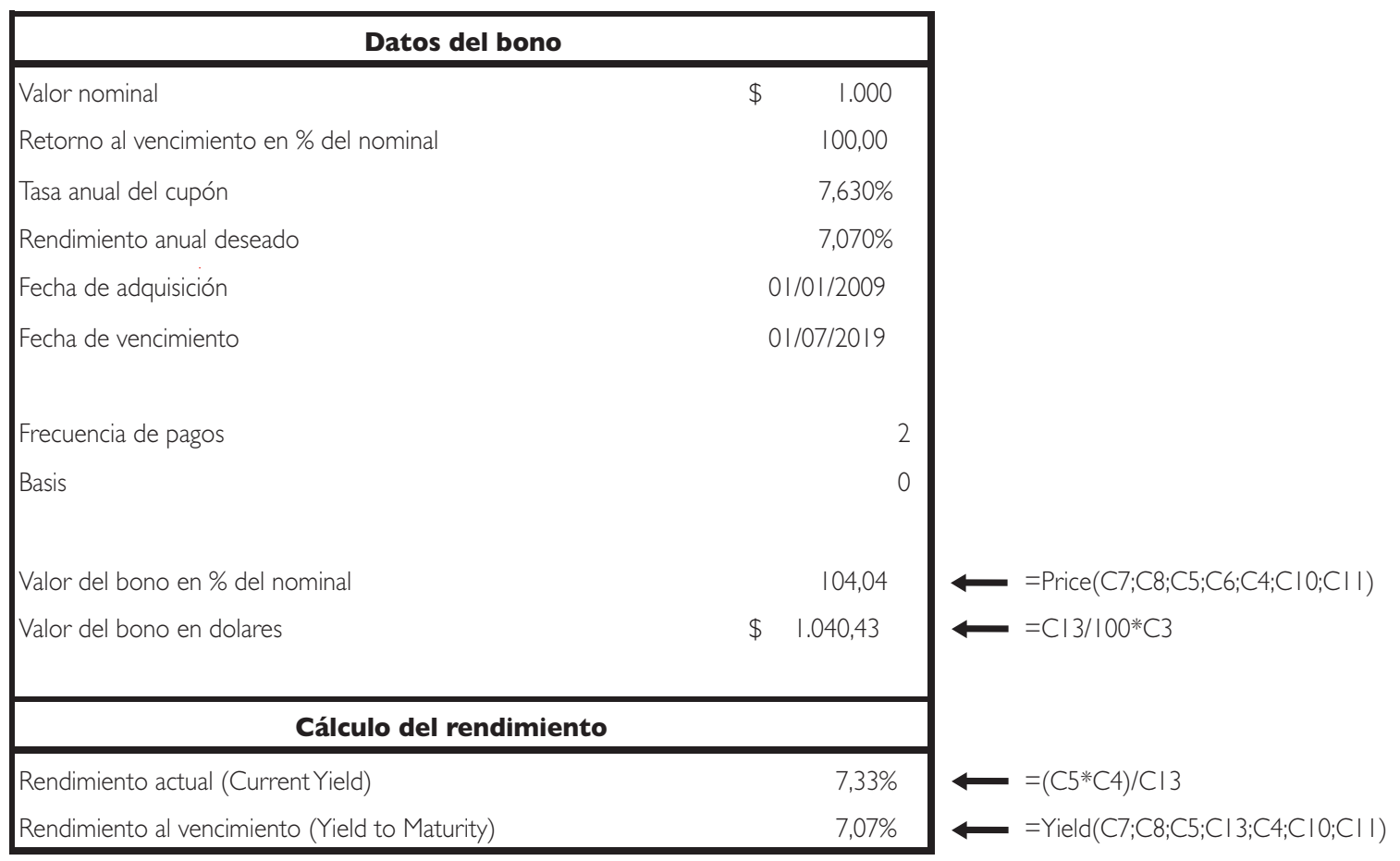

Tabla 6

Cálculo del precio de mercado al final del periodo de inversión mediante la herramienta Excel

\begin{tabular}{|c|c|c|c|}
\hline \multicolumn{3}{|l|}{ Datos del bono } & \\
\hline Valor nominal & $\$$ & 1.000 & \\
\hline Retorno al vencimiento en \% del nominal & & 100,00 & \\
\hline Tasa anual del cupón & & $7,630 \%$ & \\
\hline Rendimiento anual deseado & & $8,490 \%$ & \\
\hline Fecha de adquisición & & $101 / 2010$ & \\
\hline Fecha de vencimiento & & $107 / 2019$ & \\
\hline Frecuencia de pagos & & 2 & \\
\hline Basis & & 0 & \\
\hline Valor del bono en \% del nominal & & 94,45 & $\longleftarrow=\operatorname{Price}(\mathrm{C} 7 ; \mathrm{C} 8 ; \mathrm{C} 5 ; \mathrm{C} 6 ; \mathrm{C} 4 ; \mathrm{ClO} ; \mathrm{Cl})$ \\
\hline Valor del bono en dolares & $\$$ & 944,51 & $\longleftarrow=\mathrm{Cl} 3 / 100 * \mathrm{C} 3$ \\
\hline \multicolumn{3}{|c|}{ Cálculo del rendimiento } & \\
\hline Rendimiento actual (Current Yield) & & $8,07 \%$ & $\longleftarrow=(\mathrm{C} 5 * \mathrm{C} 4) / \mathrm{Cl} 3$ \\
\hline Rendimiento al vencimiento (Yield to Maturity) & & $8,49 \%$ & $\longleftarrow=$ Yield (C7;C8;C5;C 3;C4;Cl O;Cl I \\
\hline
\end{tabular}


Tabla 7

Cálculo del rendimiento esperado del bono mediante la herramienta Excel

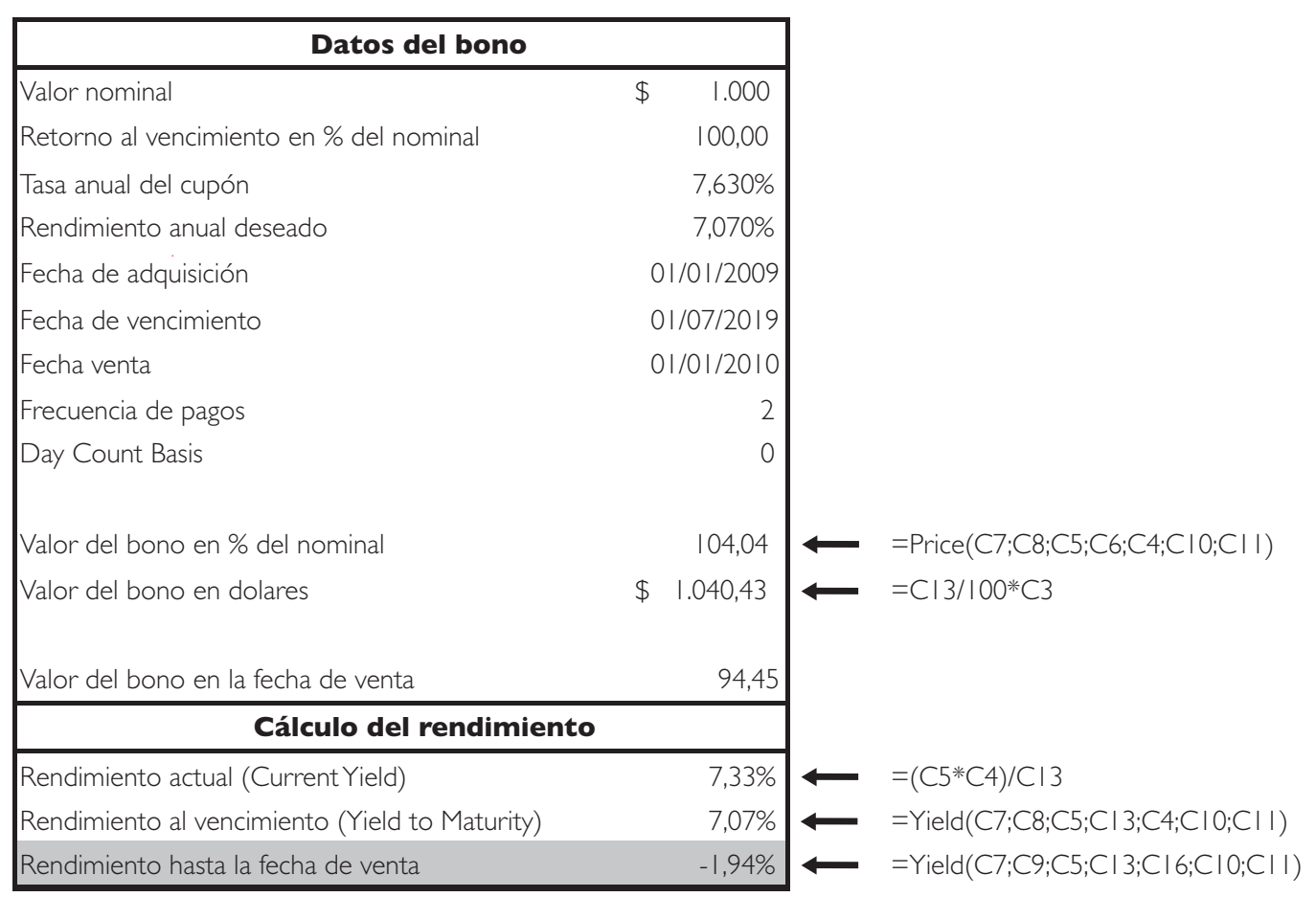

Tabla 8

Rendimiento esperado de cada bono en cada escenario

\begin{tabular}{|c|c|c|c|c|c|c|}
\hline & & \multicolumn{5}{|c|}{$r_{\text {is }}(\%)$} \\
\hline & & $r_{i 1}(\%)$ & $r_{i 2}(\%)$ & $r_{i 3}(\%)$ & $r_{i 4}(\%)$ & $r_{i 5}(\%)$ \\
\hline \multirow{8}{*}{$\begin{array}{l}\text { O } \\
i z \\
\text { m }\end{array}$} & BAC.NU & 1,89 & 4,43 & 6,91 & 9,55 & 12,12 \\
\hline & BAC.OJ & 1,76 & 4,43 & 7,04 & 9,84 & 12,55 \\
\hline & BAC.WG & 1,34 & 4,45 & 7,48 & 10,74 & 13,93 \\
\hline & CAT.LO & 2,08 & 4,50 & 6,86 & 9,40 & 11,85 \\
\hline & HBC.GBA & 1,44 & 5,24 & 8,96 & 12,99 & 16,94 \\
\hline & SPG.KD & 3,11 & 8,30 & 13,45 & 19,09 & 24,68 \\
\hline & TGT.G] & 2,74 & 5,61 & 8,43 & 11,44 & 14,39 \\
\hline & WFC.JL & 1,46 & 3,52 & 5,71 & 8,04 & $|0,3|$ \\
\hline \multirow{8}{*}{ 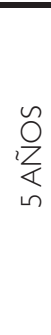 } & BAC.GEK & $-1,55$ & 4,26 & 10,02 & 16,02 & 22,62 \\
\hline & BAC.GEO & $-1,48$ & 3,90 & 9,22 & 14,73 & 20,78 \\
\hline & BAC.GER & $-1,46$ & 3,77 & 8,95 & $|4,3|$ & 20,16 \\
\hline & BAC.GFM & $-1,52$ & 3,53 & 8,83 & $|4,3|$ & 20,33 \\
\hline & CMA.HJ & $-2,26$ & $5,7 \mid$ & 13,73 & 22,25 & 31,78 \\
\hline & COF.HE & $-2,25$ & 3,51 & 9,22 & 15,48 & 21,70 \\
\hline & HBC.GCB & $-2,04$ & 3,33 & 8,64 & 14,16 & 20,21 \\
\hline & SPG.JP & $-1,62$ & 5,55 & 12,72 & 20,27 & 28,66 \\
\hline \multirow{8}{*}{ 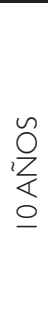 } & APA.GG & $-1,94$ & 3,84 & 10,39 & 16,79 & 23,51 \\
\hline & BAC.GWX & $-2,30$ & 3,56 & 10,19 & 16,65 & 23,41 \\
\hline & BAC.HIK & $-1,98$ & 4,63 & 12,20 & 19,68 & 27,62 \\
\hline & HBC.IKN & $-2,80$ & 4,76 & 13,45 & 22,05 & 31,20 \\
\hline & $\mathrm{HBC.IKX}$ & $-2,62$ & 4,62 & $|2,9|$ & 21,10 & 29,80 \\
\hline & $\mathrm{HBC} . \mathrm{IMH}$ & $-2,82$ & 5,85 & 15,95 & 26,08 & 37,02 \\
\hline & \begin{tabular}{|l} 
PRU.HT \\
\end{tabular} & $-2,35$ & 4,06 & 11,32 & 18,44 & 25,94 \\
\hline & UTX.GB & $-|1,8|$ & 3,33 & 9,13 & 14,77 & 20,67 \\
\hline
\end{tabular}


Realizando los tres pasos para cada uno de los 24 bonos en cada uno de los cinco escenarios, se obtienen los rendimientos esperados de cada bono en cada escenario (Tabla 8).

\section{Análisis de los resultados}

\section{I. Resultados para el modelo de cartera óptima de cada escenario independiente $\mathrm{COI}$}

De acuerdo con los datos obtenidos en el apartado 2, al observar, a modo de ejemplo las rentabilidades esperadas de los bonos en el escenario 53 , se aprecia que la mínima es 0,057 I (5,7|\%) para el bonoWFC.JL y la máxima de 0, 1595 (I5,95\%) para HBC.IMH. Al resolver el modelo $\mathrm{COI}$ con $r_{3}=0,057 \mathrm{l}$ y $C_{3}=1 \cdot 10^{6} \sqrt{0,057 \mid}=238.956,06 \$$ se obtiene como solución optima que el todo el capital debe ser invertido en el bonoWFC.JL. Esta es obviamente la única posibilidad de reparto del capital que da como rentabilidad esperada de la cartera 5,7l\%. Cualquier otra distribución de ese capital daría una rentabilidad de cartera mayor. Por tanto este sería el extremo inferior de la frontera eficiente, esto es, la cartera con el mínimo rendimiento esperado dentro de frontera eficiente. Si se resuelve el modelo COI para $r_{3}=0,1595$ y $C_{3}=1 \cdot 10^{6} \sqrt{0,1595}=399.394,51 \$$, se obtendrá como resultado que se debe invertir todo el capital en HBC.IMH y esa es la única cartera que tendrá esa rentabilidad esperada. Esta sería la cartera que estaría en el extremo superior de la frontera eficiente. Para cualquier otra rentabilidad esperada de carte- ra $r_{3} \in\left(0^{\prime} 057,0^{\prime}\right.$ I 373) existirán numerosas posibilidades de reparto del capital entre los 24 bonos de manera que se obtenga esa rentabilidad esperada. De entre todas estas posibilidades de reparto del capital para cada $r_{3}$, el modelo proporciona la solución con el riesgo mínimo definido como la varianza de la cartera. Al resolver el modelo para varios valores de $r_{3}$ dentro de este intervalo, se obtiene la frontera de carteras eficientes para el escenario s3, esto es, el conjunto de carteras que para cada rentabilidad tienen la mínima varianza (mínimo riesgo). La frontera eficiente para este escenario se muestra en la Figura 2.

Del conjunto de carteras eficientes para el escenario s3, representadas en la Figura 2 por su frontera eficiente, se ha resaltado la que se ha definido como cartera óptima del escenario y que es aquella cuyo rendimiento esperado es igual a la media de los rendimientos esperados de todos los bonos en ese escenario, la cual servirá como referencia en el modelo coordinado:

$$
r_{3}=\frac{\sum_{i=1}^{24} r_{i 3}}{24}=10,07 \%
$$

El capital invertido en esta cartera es

$$
c_{3}=1 \cdot 10^{6} \sqrt{0,1007}=317.352,33 \$
$$

y el riesgo asociada a la misma 5,99 × 109 .

Esto mismo se ha de realizar para cada escenario, tomando como cartera óptima de cada escenario la

Figura 2

Frontera de carteras eficientes para el escenario s3

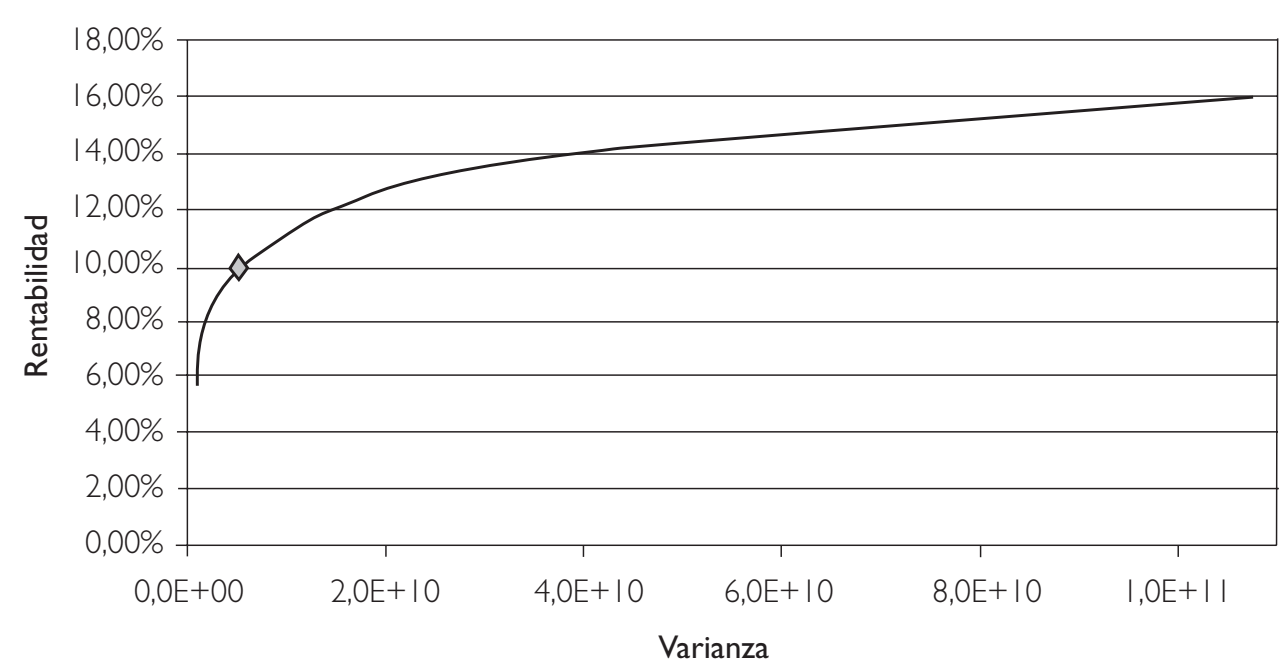


cartera, dentro del conjunto de carteras que forman la frontera eficiente, cuyo rendimiento esperado sea la media de los rendimientos esperados de todos los bonos para ese escenario y la varianza sea la mínima posible. Tan solo se ha practicado una salvedad que será para el caso del escenario sl. De hecho, el rendimiento esperado para los bonos de 5 y 10 años en este escenario es negativo (Tabla 7), y al tomar la media de todos los rendimientos proporciona un valor negativo. Ya que conscientemente no se invertiría en un bono que se ha estimado que tendrá rendimiento negativo, se tomará como valor del rendimiento esperado de la cartera óptima del escenario sl la media de los rendimientos positivos.

A continuación se muestra una tabla con la composición de las carteras óptimas de cada escenario, así como el rendimiento esperado, capital a invertir y riesgo esperado de cada una de ellas (Tabla 9).

\subsection{Resultados para el modelo de error coordinado de la inversión en el activo respecto al escenario s según el modelo MM}

Una vez obtenidas las carteras optimas de cada escenario y el capital a invertir en cada una de ellas, se incorpora toda esta información al modelo coordinado (Modelo MM) que proporcionará la composición de la cartera real y el capital a invertir en ella, de manera que se minimice el error coordinado respecto a cada uno de los escenarios.

Como primer supuesto para el análisis se ha supuesto equiprobabilidad de escenarios. Es decir, $P_{s}=0,2$ (20\%) para los cinco escenarios, $C_{\min }=C_{1}=$ $|40.623,6| \$$ y $C_{\max }=C_{5}=466.449,35 \$$.

Luego la composición de cartera solución del Modelo MM quedará según se muestra en la Tabla 10.

Tabla 9

Carteras óptimas de cada escenario

\begin{tabular}{|c|c|c|c|c|c|c|}
\hline & & & & & & \\
\hline & & sl & s2 & s3 & s4 & s5 \\
\hline & $r_{s}$ & $1,98 \%$ & $4,53 \%$ & $10,07 \%$ & $15,76 \%$ & $21,76 \%$ \\
\hline & $C_{s}$ & $\$ 140.623,6 \mid$ & $\$ 212.808,60$ & $\$ 317.352,33$ & $\$ 396.957,18$ & $\$ 466.449,35$ \\
\hline & $L\left(x_{s}\right)$ & $4|2.107 .7| 4,58$ & $8|5.339 .865,5|$ & $5.991 .862 .077,55$ & $8.287 .578 .555,80$ & $9.879 .275 .128,02$ \\
\hline & \multicolumn{6}{|c|}{$x_{s}$} \\
\hline \multirow{8}{*}{ 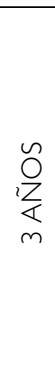 } & BAC.NU & 0,00 & 0,00 & 0,00 & 0,00 & 0,00 \\
\hline & BAC.OJ & $\$ 70.020,54$ & $\$ 207.378,41$ & 0,00 & 0,00 & 0,00 \\
\hline & BAC.WG & 0,00 & 0,00 & 0,00 & 0,00 & 0,00 \\
\hline & CAT.LO & $\$ 58.492,99$ & 0,00 & 0,00 & 0,00 & 0,00 \\
\hline & HBC.GBA & 0,00 & 0,00 & 0,00 & 0,00 & 0,00 \\
\hline & SPG.KD & 0,00 & $\$ 5.430,19$ & $\$ 85.262,68$ & 0,00 & 0,00 \\
\hline & TGT.GJ & $\$ 12.110,08$ & 0,00 & 0,00 & 0,00 & 0,00 \\
\hline & WFC.JL & 0,00 & 0,00 & 0,00 & 0,00 & 0,00 \\
\hline \multirow{8}{*}{ 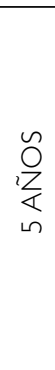 } & BAC.GEK & 0,00 & 0,00 & 0,00 & 0,00 & 0,00 \\
\hline & BAC.GO & 0,00 & 0,00 & 0,00 & 0,00 & 0,00 \\
\hline & BAC.GER & 0,00 & 0,00 & 0,00 & 0,00 & 0,00 \\
\hline & BAC.GFM & 0,00 & 0,00 & $\$ 232.089,65$ & $\$ 300.548,53$ & $\$ 386.5|4,6|$ \\
\hline & CMA.HJ & 0,00 & 0,00 & 0,00 & 0,00 & 0,00 \\
\hline & COF.HE & 0,00 & 0,00 & 0,00 & 0,00 & 0,00 \\
\hline & HBC.GCB & 0,00 & 0,00 & 0,00 & 0,00 & 0,00 \\
\hline & SPG.JP & 0,00 & 0,00 & 0,00 & $\$ 96.408,64$ & $\$ 79.934,75$ \\
\hline \multirow{8}{*}{ 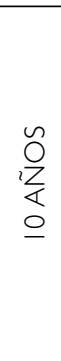 } & APA.GG & 0,00 & 0,00 & 0,00 & 0,00 & 0,00 \\
\hline & BAC.GWX & 0,00 & 0,00 & 0,00 & 0,00 & 0,00 \\
\hline & BAC.HK & 0,00 & 0,00 & 0,00 & 0,00 & 0,00 \\
\hline & HBC.IKN & 0,00 & 0,00 & 0,00 & 0,00 & 0,00 \\
\hline & HBC.IKX & 0,00 & 0,00 & 0,00 & 0,00 & 0,00 \\
\hline & $\mathrm{HBC} . \mathrm{IMH}$ & 0,00 & 0,00 & 0,00 & 0,00 & 0,00 \\
\hline & PRU.HT & 0,00 & 0,00 & 0,00 & 0,00 & 0,00 \\
\hline & UTX.GB & 0,00 & 0,00 & 0,00 & 0,00 & 0,00 \\
\hline
\end{tabular}


Tabla 10

Cartera coordinada del Modelo MM

\begin{tabular}{|c|c|c|}
\hline & & $x_{i}$ \\
\hline \multirow{8}{*}{ 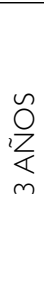 } & BAC.NU & 7,64 \\
\hline & BAC.OJ & $246.700,49$ \\
\hline & BAC.WC & $4.289,94$ \\
\hline & CAT.LO & $3.378,30$ \\
\hline & HBC.GBA & $5.670,43$ \\
\hline & SPG.KD & $9.533,27$ \\
\hline & TGT.GJ & 78,42 \\
\hline & WFC.JL & 110,70 \\
\hline \multirow{8}{*}{ 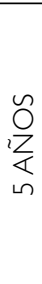 } & BAC.GEK & $6.526,57$ \\
\hline & BAC.GEO & $6.962,83$ \\
\hline & BAC.GER & $6.634,08$ \\
\hline & BAC.GFM & $47.545,01$ \\
\hline & CMA.HJ & 6,68 \\
\hline & COF.HE & $6.758,55$ \\
\hline & HBC.GCB & $3.731,88$ \\
\hline & SPG.JP & $10.602,79$ \\
\hline \multirow{8}{*}{$\begin{array}{l}\sim \\
\stackrel{0}{Z} \\
\stackrel{1}{<} \\
0\end{array}$} & APA.GG & $7.564,83$ \\
\hline & BAC.GWX & $5.740,67$ \\
\hline & BAC.HIK & $8.270,86$ \\
\hline & $\mathrm{HBC} . \mathrm{IKN}$ & $7.709,59$ \\
\hline & HBC.IKX & $6.617,19$ \\
\hline & HBC.IMH & $11.006,78$ \\
\hline & PRU.HT & $8.651,41$ \\
\hline & UTX.GB & $6.281,20$ \\
\hline & $C(x)$ & $\$ 420.446,12$ \\
\hline & $L(x)$ & $8.257 .308 .969,00$ \\
\hline
\end{tabular}

En la Figura 3 se muestran los errores coordinados y los errores coordinados ponderados respecto a cada uno de los escenarios para esta solución.
A continuación (Tabla I I) se compara la cartera óptima de cada escenario con la cartera coordinada, en el caso de que se produjera dicho escenario.

Luego para una distribución equiprobable de escenarios se obtiene una cartera coordinada en la que el beneficio esperado es mayor que el de la cartera óptima en los escenarios centrales y menor en los extremos. El error coordinado para los escenarios extremos es el mismo. La cartera coordinada está más diversificada que las carteras óptimas donde el capital a invertir se concentraba en 2 o 3 títulos. El capital a invertir en la cartera coordinada $(420.446,12$ \$) está más próximo a $C_{\max }(466.449,35 \$)$ que a $C_{\min }$ ( $40.623,6$ | \$). Por último, el riesgo de la cartera coordinada $(8.257 .308 .969,00)$ está más próximo al riesgo máximo $\left[L\left(x_{5}\right)=9.879 .275 .128,02\right]$ que al mí$\operatorname{nimo}\left(L\left(x_{1}\right)=4|2 \cdot 107.7| 4,58\right)$.

\subsection{Análisis de sensibilidad: Modelo MM}

Con objeto de llevar a cabo un análisis de sensibilidad ante distintas probabilidades de ocurrencia de escenarios, se ha analizado la influencia que tienen las probabilidades asignadas a cada escenario, en la solución final que proporciona el modelo. Para ello se considera otra distribución de probabilidades en la que el escenario más probable es el escenario sl. Se analiza si esto mejora los resultados obtenidos por la cartera óptima en este escenario y que in-

Figura 3

Errores coordinados de la cartera coordinada del modelo MM

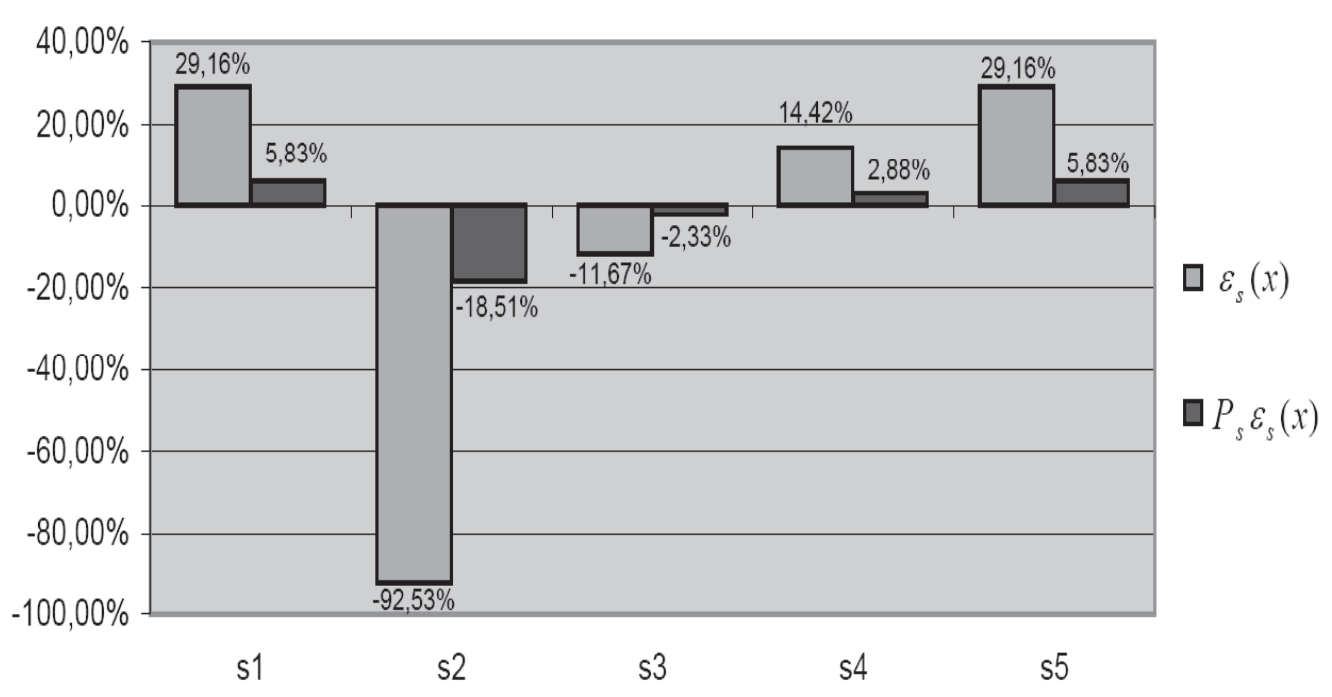


Tabla I I

Comparación de las carteras óptimas de cada escenario con las carteras coordinadas
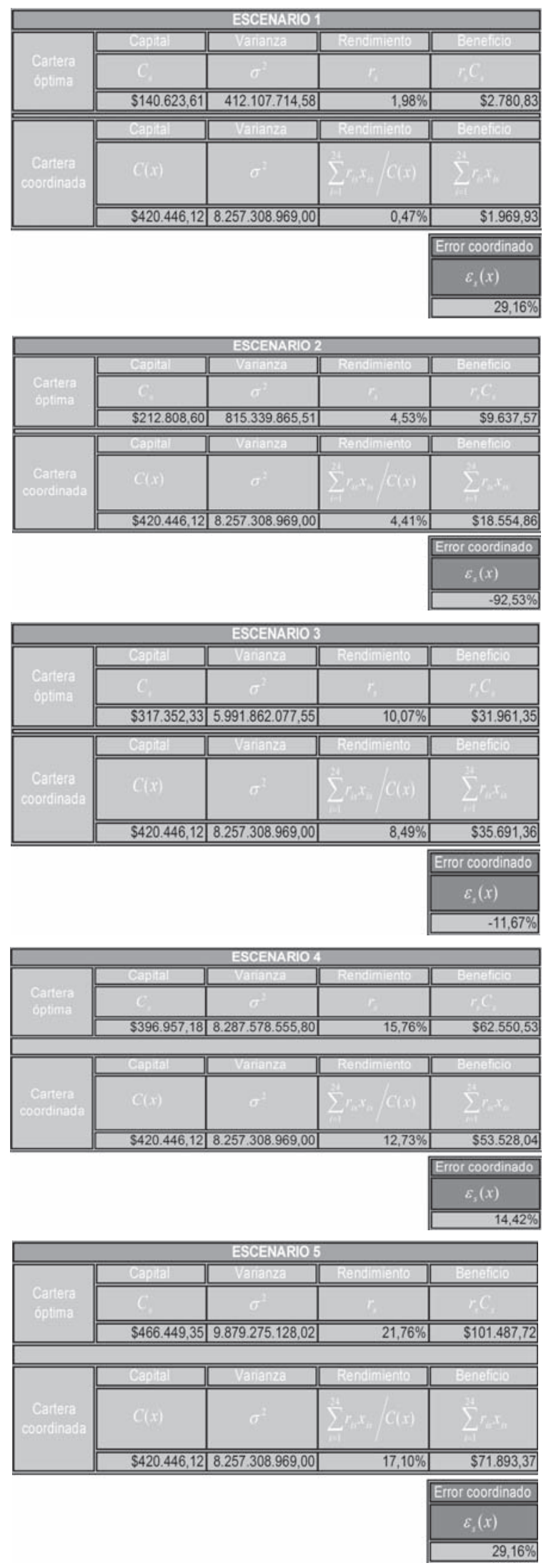

fluencia tiene sobre el resto de escenarios. Se han tomado como distribución de probabilidades el 40\% para $S_{1}, 30 \%$ para $S_{2}, 15 \%$ para $S_{3}, 10 \%$ para $S_{4}$ y $5 \%$ para $S_{5}$. Con todo ello, la composición de la cartera que proporciona el Modelo MM es la siguiente (Tabla 12):

Tabla 12

Cartera coordinada del Modelo MM con distribución asimétrica de probabilidades

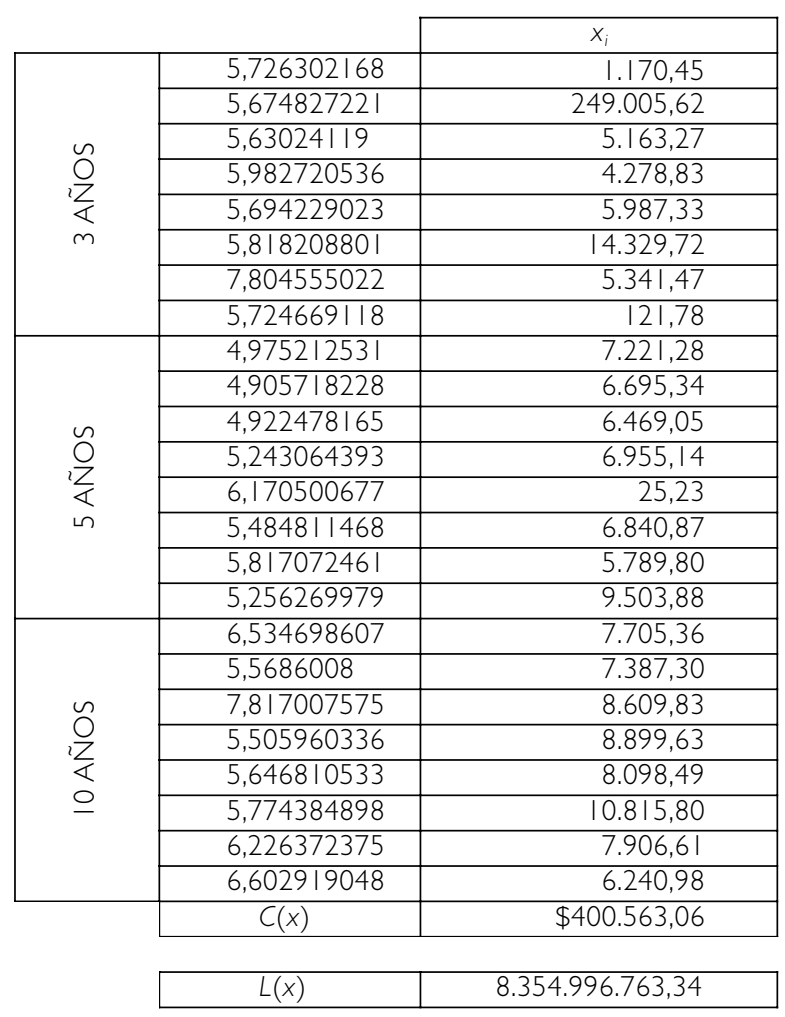

Al aumentar la probabilidad de ocurrencia del escenario I, el capital a invertir disminuye, acercándose un poco al capital de la cartera óptima de este escenario, aunque manteniéndose aún entre los capitales de los escenarios 4 y 5 . El riesgo se mantiene en un nivel parecido, por debajo del riesgo del último escenario como impone la cuarta restricción.

Los errores coordinados y los errores coordinados ponderados, respecto a cada uno de los escenarios en el óptimo se representan en la Figura 4.

A partir estos datos pueden hacerse varias observaciones importantes. Por un lado, el error coordinado respecto al escenario I ahora es negativo. Esto quiere decir que la cartera coordinada tiene un beneficio esperado mayor que el óptimo de este escenario, que ahora es el más probable. Los errores 
Figura 4

Errores coordinados de la cartera coordinada del Modelo MM con distribución asimétrica de probabilidades

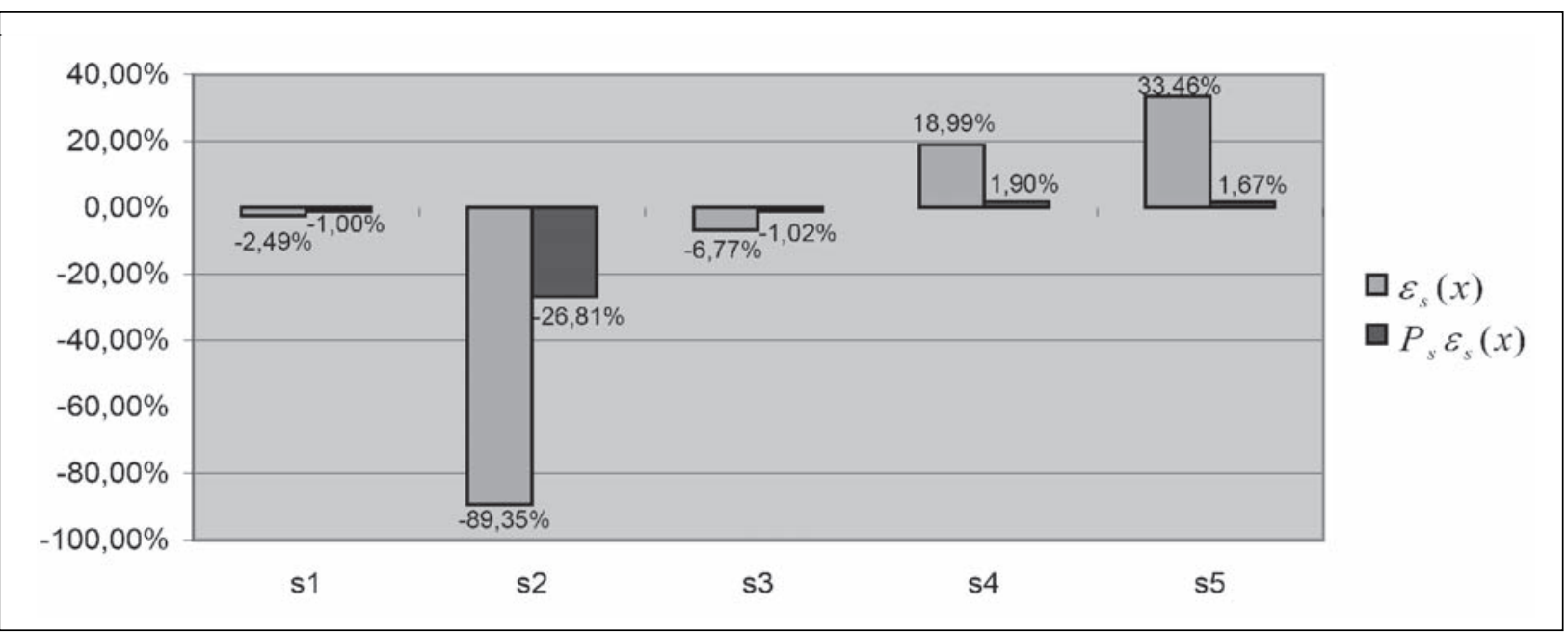

coordinados respecto a los escenarios 2 y 3 siguen siendo negativos aunque no tanto como en el caso anterior, especialmente para el escenario 3. Para los escenarios 4 y 5 los errores coordinados siguen siendo positivos y además mayores que en el caso anterior, al ser ahora menores las probabilidades de ocurrencia de ambos. El máximo error coordinado se produce para el último escenario, el menos probable, con un 33,46\%. Sin embargo, al ser la probabilidad de este escenario la mitad que la del escenario 4, es en este último donde $P_{s} \varepsilon_{s}(x)$ toma el máximo valor positivo (I,90\%) siendo éste, por tanto, el valor de la función objetivo en el óptimo.
Por tanto, puede decirse que el modelo MM responde favorablemente a esta nueva distribución de probabilidades de los escenarios acercándose en términos de beneficio esperado al óptimo del escenario más probable y alejándose de los menos probables.

\subsection{Resultados para el modelo de error coordinado de la inversión en el activo respecto al escenario s según el modelo MAD}

A continuación se acomete el estudio empleando la función objetivo asociada al Modelo MAD, y considerando inicialmente condiciones de equiprobabili-

Figura 5

Errores coordinados de la cartera coordinada del Modelo MAD

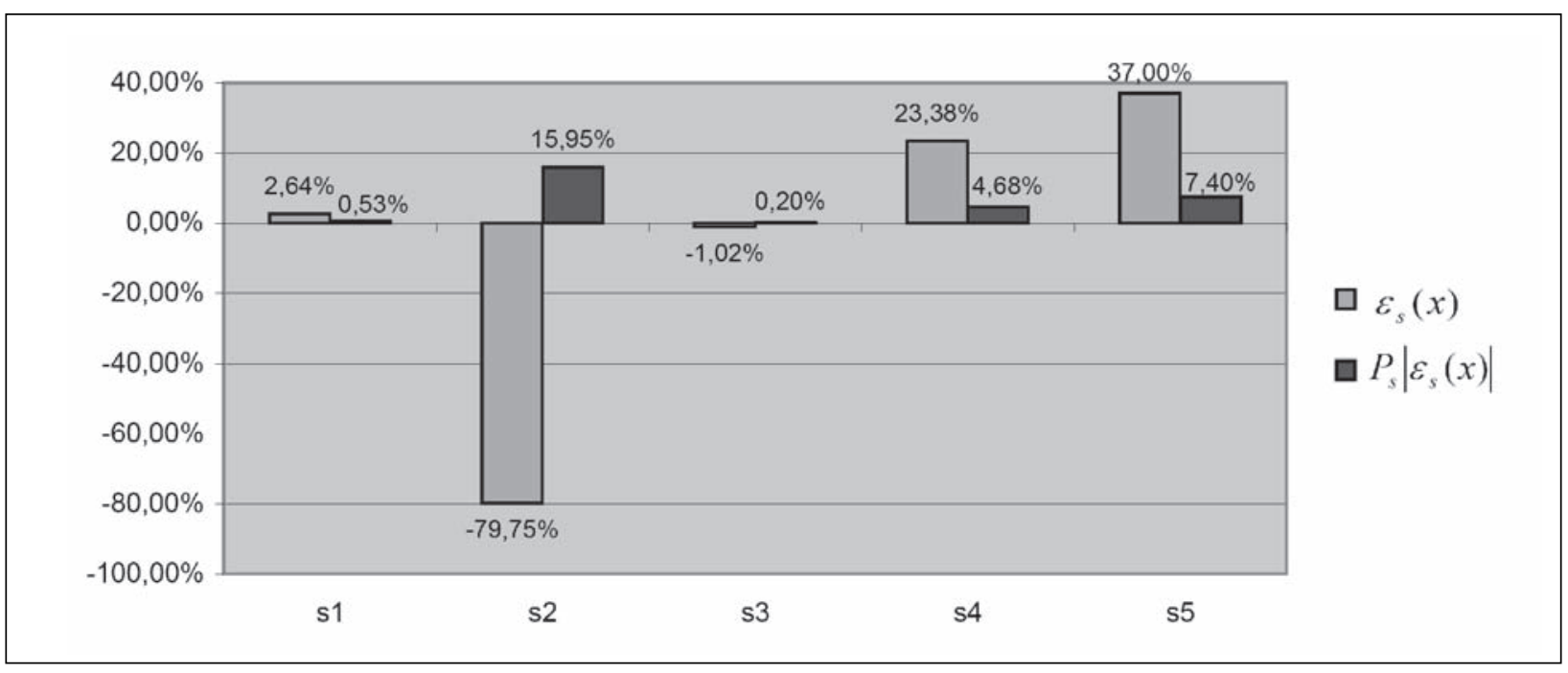


Tabla 13

Cartera coordinada del Modelo MAD

\begin{tabular}{|c|c|c|}
\hline & \multirow[b]{2}{*}{$x_{\mathrm{i}}$} \\
\hline & & \\
\hline \multirow{8}{*}{ 柋 } & BAC.NU & $6.604,92$ \\
\hline & BAC.OJ & $245.347,92$ \\
\hline & BAC.WG & $2.680,99$ \\
\hline & CAT.LO & $2.120,29$ \\
\hline & HBC.GBA & $3.770,86$ \\
\hline & SPG.KD & $7.141,82$ \\
\hline & TGT.GJ & $5.241,95$ \\
\hline & WFC.JL & $1.319,89$ \\
\hline \multirow{8}{*}{ 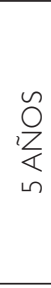 } & BAC.GEK & $3.6|5,3|$ \\
\hline & BAC.GEO & $4.234,40$ \\
\hline & BAC.GER & $3.975,30$ \\
\hline & BAC.GFM & $45.016,70$ \\
\hline & CMA.HJ & $1.350,58$ \\
\hline & COF.HE & $3.870,73$ \\
\hline & HBC.GCB & $8.163,60$ \\
\hline & SPG.JP & $7.092,50$ \\
\hline \multirow{8}{*}{$\begin{array}{l}\text { Õ } \\
\text { iz } \\
\text { ○ } \\
0\end{array}$} & APA.GG & $8.133,64$ \\
\hline & BAC.GWX & $6.053,06$ \\
\hline & BAC.HIK & $5.090,13$ \\
\hline & HBC.HIK & $2.009,68$ \\
\hline & HBC.IKX & $3.153,01$ \\
\hline & $\mathrm{HBC} . \mathrm{IMH}$ & $7.015,33$ \\
\hline & PRU.HT & $5.533,57$ \\
\hline & UTX.GB & $7.200,62$ \\
\hline & $C(x)$ & $\$ 395.736,77$ \\
\hline & $L(x)$ & $6.090 .337 .539,99$ \\
\hline
\end{tabular}

Tabla 14

Cartera coordinada del Modelo MAD con distribución asimétrica de probabilidades

\begin{tabular}{|c|c|c|}
\hline & & $x_{i}$ \\
\hline \multirow{8}{*}{$\begin{array}{l}\text { On } \\
\stackrel{i}{Z} \\
\text { m }\end{array}$} & BAC.NU & $2.627,04$ \\
\hline & BAC.OJ & $200.950,33$ \\
\hline & BAC.WG & $2.638,22$ \\
\hline & CAT.LO & $2.604,50$ \\
\hline & HBC.GBA & $2.648,95$ \\
\hline & SPG.KD & $4.050,67$ \\
\hline & TGT.GJ & $9.078,04$ \\
\hline & WFC.JL & $10.727,22$ \\
\hline \multirow{8}{*}{ 帒 } & BAC.GEK & $4.947,46$ \\
\hline & BAK.GEO & $4.834,47$ \\
\hline & BAK.GER & $4.384,03$ \\
\hline & BAK.GFM & $6.604,74$ \\
\hline & CMA.HJ & $5.348,88$ \\
\hline & COF.HE & $5.075,34$ \\
\hline & HBC.GCB & $5.231,27$ \\
\hline & SPG.JP & $3.808,91$ \\
\hline \multirow{8}{*}{ 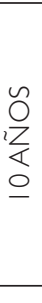 } & APA.GG & $3.739,24$ \\
\hline & BAC.GWX & $3.503,44$ \\
\hline & BAC.HIK & $3.556,09$ \\
\hline & HBC.IKN & $4.408,31$ \\
\hline & HBC.IKX & $4.108,26$ \\
\hline & HBC.IMH & $1.674,02$ \\
\hline & PRU.HT & $1.308,70$ \\
\hline & UTX.GB & $4.894,29$ \\
\hline & $C(x)$ & $\$ 302.752,42$ \\
\hline & $L(x)$ & $3.987 .875 .973,40$ \\
\hline
\end{tabular}

dad de escenarios. Así, la composición de cartera que proporciona el Modelo MAD será la reflejada en la Tabla 13.

Los errores coordinados y los coordinados ponderados, respecto a cada escenario, están representados en la Figura 5.

\subsection{Análisis de sensibilidad: Modelo MAD}

Para observar el grado de robustez o sensibilidad de la propuesta ante posibles modificaciones en los datos de entrada relativos a la probabilidad de ocurrencia de los escenarios, se ha considerado el problema con distribuciones de probabilidades del 40\% para $S_{1}, 30 \%$ para $S_{2}, 15 \%$ para $S_{3}, 10 \%$ para $S_{4}$ y $5 \%$ para $S_{5}$, con lo que se obtiene la siguiente cartera coordinada (Tabla |4).

En la Figura 6 se muestran los errores coordinados y los errores coordinados ponderados de esta solución respecto a cada escenario.

\section{Análisis comparado de los resultados de los modelos}

Una vez estudiadas y comparadas las soluciones que proporciona cada modelo para el caso de escenarios equiprobables, se obtiene la Tabla 15 (véase p. 84).

Analizando los resultados obtenidos para cada escenario, así como la solución de los modelos coordinados (para el caso de distribución de escenarios asimétrica), se argumenta que en el Modelo MM el capital y el riesgo coordinados son mayores que en el Modelo MAD. El beneficio esperado (como suma del beneficio esperado en cada escenario) es también mayor para dicho modelo. Por todo ello, el rendimiento es ligeramente superior para la cartera solución del Modelo MM. En cambio, la diferencia entre los errores coordinados máximo y mínimo es ligeramente inferior para el Modelo MAD, siendo, además, la suma de los errores coordinados respecto a cada uno de los escenarios, más negativa para el Modelo MM. Esto último da una idea de la desviación del beneficio esperado de la cartera coordinada respecto al beneficio esperado de la cartera óptima. Una vez obtenido el error coordinado (en valor absoluto), se ha definido el índice de robustez (12), el cual se ha basado en la variación del beneficio esperado del proyecto de inversión al óptimo de cada escenario. Analizados los cuadros comparativos de ambos modelos, se puede determinar que el índice de robustez es ma- 
Figura 6

Errores coordinados de la cartera coordinada del modelo MAD con distribución asimétrica de probabilidades

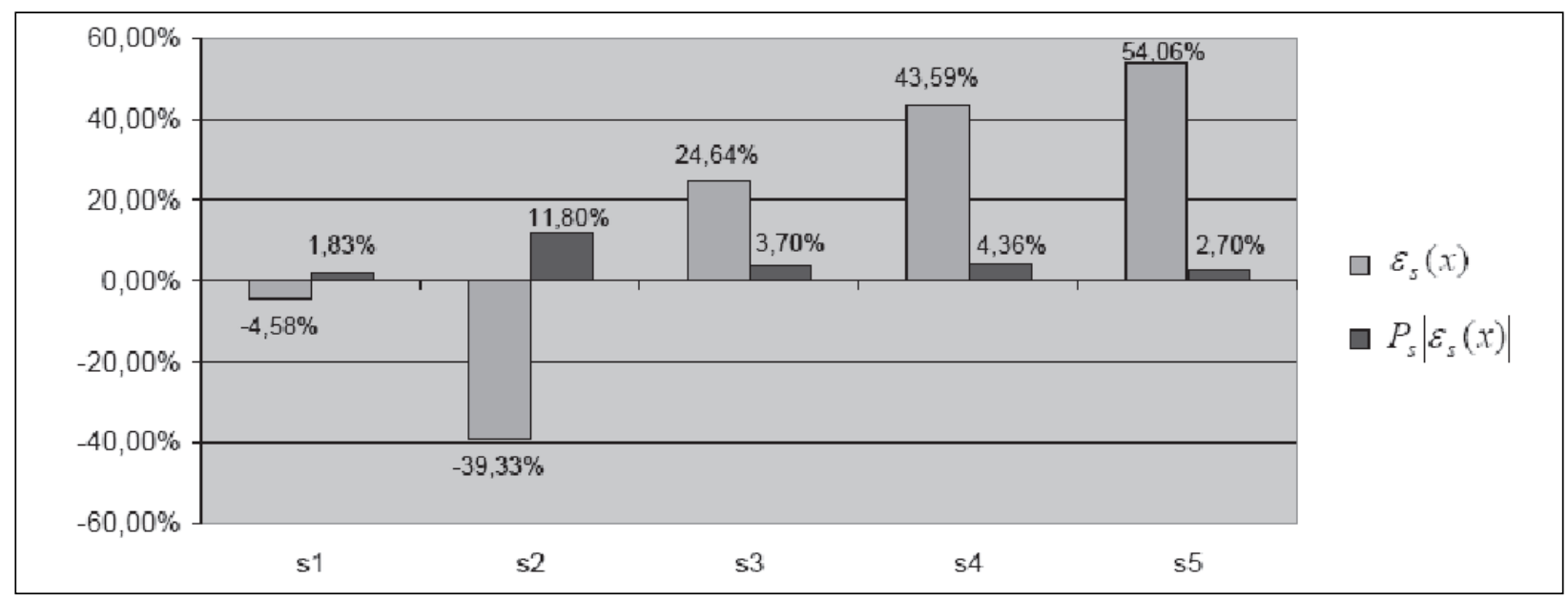

yor para el Modelo MAD. El índice de robustez expresa, por tanto, lo cerca que está cada uno de los dos modelos del modelo ideal. Un modelo coordinado ideal, cuya cartera coordinada tuviera un beneficio esperado en cada escenario igual al beneficio esperado de la cartera óptima de cada uno de ellos, tendría un índice de robustez igual a I ( I00\%), ya que todos sus errores coordinados serían cero.

Para el caso de distribución asimétrica de probabilidades de escenario se obtendría la Tabla 16 (véase siguiente página).

En el caso de que la distribución de probabilidades de escenarios sea asimétrica, y para el Modelo MAD, el capital a invertir en la cartera coordinada se acerca de manera más rápida al capital a invertir en el óptimo del escenario más probable. De hecho, el Modelo MAD responde más favorablemente a esta nueva distribución de probabilidades proporcionando una varianza con un menor nivel de riesgo. El rendimiento medio esperado por escenario para la cartera coordinada del Modelo MM sigue siendo mayor para esta distribución de probabilidades. Al igual que en la distribución equiprobable, la media de los errores coordinados ponderados, en valor absoluto, es menor para el Modelo MAD. Por el mismo motivo que en el caso anterior, el índice de robustez para el Modelo MAD sigue siendo mayor que para el Modelo MM.

\section{Conclusiones}

En el presente trabajo se han estudiado y analizado los modelos de optimización robusta como herramientas eficientes para la gestión del riesgo de títulos de renta fija.
Se ha presentado un problema consistente en la composición de carteras de inversión de renta fija partiendo de una dotación inicial indeterminada.

La parte teórica del trabajo se complementa con el estudio y resolución del modelo de optimización por escenarios (aplicado al problema de selección de cartera y partiendo de una dotación inicial indeterminada). Para ello, se han utilizado los modelos MM y MAD, habiéndose acometido un análisis de sensibilidad ante las distintas posibilidades de ocurrencia de los diferentes escenarios para cada uno de los modelos.

A la vista de los resultados presentados en las secciones 3 y 4 , se aprecia que, independientemente de que la distribución de probabilidades de escenarios sea simétrica o asimétrica, el modelo MAD es el que se comporta de una forma más robusta.

Es importante destacar que el riesgo coordinado ponderado (1 3), para el escenario más probable (s l), es negativo para ambos modelos, siendo mejor (más negativo) para el modelo MAD.

De forma genérica, se puede afirmar que los modelos desarrollados para la minimización del impacto del riesgo sobre las inversiones en el mercado de renta fija, son soluciones para prevenir los posibles quebrantos derivados del mismo, aportando una gran solidez a la toma de decisiones en entidades de inversión.

Por último, los resultados obtenidos son mejores que los que se hubiesen obtenido utilizando estrategias inmunizadores ya que este tipo de estrategias se usan para combinar el riesgo de los tipos de interés 
Tabla 15

Comparativa de los resultados de los dos modelos

\begin{tabular}{|c|c|c|c|}
\hline & \multirow{2}{*}{$\begin{array}{l}\text { MODELO MM } \\
\text { Uin } \quad \varepsilon \\
P_{s} \varepsilon_{s}(x) \leq \varepsilon\end{array}$} & \multirow{2}{*}{$\begin{array}{l}\text { MODELO MAD } \\
\text { Min } \varepsilon=\sum_{s=1}^{S} P_{s}\left|\varepsilon_{s}\right|\end{array}$} \\
\hline & & & \\
\hline (1) & $C(x)$ & $\$ 420.446,12$ & $\$ 395.736,77$ \\
\hline (2) & $L(x)$ & $8.257 .308 .969,00$ & $6.090 .337 .539,99$ \\
\hline (3) & $\sum_{s=1}^{5} \sum_{i=1}^{24} r_{i s} x_{i s}$ & $\$ 181.637,56$ & $\$ 164.174,45$ \\
\hline (4) & $\sum_{s=1}^{5} \sum_{i=1}^{24} r_{i s} x_{i s} / 5$ & $\$ 36.327,51$ & $\$ 32.834,89$ \\
\hline (5) & $\frac{\sum_{s=1}^{5} \sum_{i=1}^{24} r_{i s} x_{i s} / 5}{C(x)}$ & $8,64 \%$ & $8,30 \%$ \\
\hline (6) & $P_{s} \varepsilon_{s}(x) \max$ & $5,83 \%$ & $7,40 \%$ \\
\hline (7) & $P_{s} \varepsilon_{s}(x) \min$ & $-18,51 \%$ & $-15,95 \%$ \\
\hline (8) & $P_{s} \varepsilon_{s}(x) \max -P_{s} \varepsilon_{s}(x) \min$ & $24,34 \%$ & $23,35 \%$ \\
\hline (9) & $\sum_{s=1}^{5} P_{s} \varepsilon_{s}(x)$ & $-6,29 \%$ & $-3,55 \%$ \\
\hline (10) & $\sum_{s=1}^{5} P_{s}\left|\varepsilon_{s}(x)\right|$ & $35,39 \%$ & $28,76 \%$ \\
\hline (11) & $\sum_{s=1}^{5} P_{s} \varepsilon_{s}(x) \mid / 5$ & $7,08 \%$ & $5,75 \%$ \\
\hline (12) & $\Re=1-\sum_{s=1}^{5} P_{s} \mid \varepsilon_{s}(x) / 5$ & $92,92 \%$ & $94,25 \%$ \\
\hline
\end{tabular}

de una cartera de activos frente a un flujo de activos, con idea de que la exposición al riesgo de la misma sea nula. Por ello, para seguir una estrategia inmunizadora es necesario un reajuste periódico de la cartera para mantener su duración igual al Riesgo de Reinversión (HPI), excepto cuando se estén utilizando bonos de cupón cero. El problema que se presenta es que mantener la cartera inmunizada a lo largo del HPI implica una reestructuración continua de la cartera, debido a la evolución temporal de la duración, lo que supone unos costes de transacción elevados, reduciéndose así la rentabilidad de la cartera.

A todo ello hay que añadir que otro problema de las estrategias inmunizadoras es el riesgo de reinversión al que se está sujeto.
Tabla 16

Comparativa de los resultados de los dos modelos con distribución asimétrica de probabilidades

\begin{tabular}{|c|c|c|c|}
\hline & \multirow{2}{*}{$\begin{array}{l}\text { MODELO MM } \\
\text { lin } \varepsilon \\
P_{s} \varepsilon_{s}(x) \leq \varepsilon\end{array}$} & \multirow{2}{*}{$\begin{array}{l}\text { MODELO MAD } \\
\text { Min } \varepsilon=\sum_{s=1}^{5} P_{s} \mid \varepsilon_{s}(x)\end{array}$} \\
\hline & & & \\
\hline (1) & $C(x)$ & $\$ 400.563,06$ & $\$ 302.752,42$ \\
\hline (2) & $L(x)$ & $8.354 .996 .763,34$ & $3.987 .875 .973,40$ \\
\hline (3) & $\sum_{s=1}^{5} \sum_{i=1}^{24} r_{i s} x_{i s}$ & $\$ 173.427,43$ & $\$ 122.335,75$ \\
\hline (4) & $\sum_{s=1}^{5} \sum_{i=1}^{24} r_{i s} x_{i s} / 5$ & $\$ 34.685,49$ & $\$ 24.467,15$ \\
\hline (5) & $\frac{\sum_{s=1}^{5} \sum_{i=1}^{24} r_{i s} x_{i s} / 5}{C(x)}$ & $8,66 \%$ & $8,08 \%$ \\
\hline (6) & $P_{s} \varepsilon_{s}(x) \max$ & $1,67 \%$ & $4,36 \%$ \\
\hline (7) & $P_{s} \varepsilon_{s}(x) \min$ & $-26,81 \%$ & $-11,80 \%$ \\
\hline (8) & $P_{s} \varepsilon_{s}(x) \max -P_{s} \varepsilon_{s}(x) \mathrm{min}$ & $28,48 \%$ & $16,16 \%$ \\
\hline (9) & $\sum_{s=1}^{5} P_{s} \varepsilon_{s}(x)$ & $-25,24 \%$ & $-2,87 \%$ \\
\hline (10) & $\sum_{s=1}^{5} P_{s}\left|\varepsilon_{s}(x)\right|$ & $32,39 \%$ & $24,39 \%$ \\
\hline (11) & $\sum_{s=1}^{5} P_{s}\left|\varepsilon_{s}(x)\right| / 5$ & $6,48 \%$ & $4,88 \%$ \\
\hline (12) & $\Re=1-\sum_{s=1}^{5} P_{s} \varepsilon_{s}(x) / 5$ & $93,52 \%$ & $95,12 \%$ \\
\hline (13) & $P_{1} \varepsilon_{1}(x)$ & $-1,00 \%$ & $-1,83 \%$ \\
\hline
\end{tabular}

\section{Referencias}

BAI, D.,CARPENTER, T AND MULVEY, J. ( 1 997). «Making a Case for Robust Optimization Models». Management Science 43(7), pp. 895-907.

CANÓS, M. J.y VENTURA, M (1999). «Un modelo de cartera con incertidumbre». Actas de las VII Jornadas de ASEPUMA, pp. 168- 177.

CHOPRA, ZIEMBA. (1993). «The Effect of Errors in Means, Variances, and Covariances on Optimal Portfolio Choice». The Journal of Portfolio Management 19(2), pp. 6-II.

DEMBO, R.S. (|99|). «Scenario Inmunization». Annals of Operations Research 30(I), pp. 63-80.

ESCUDERO, L. (1995). «Robust portfolios for mortgagebacked securities. Quantitative Methods». Al and Supercomputers in Finance (Unicom, Londres), pp. 20 I-228. 
GOLUB, B., HOLMER, M., MCKENDALL, R., POHLMAN, L. y ZENIOS, S.A. (1994). «A stochastic programming models for money management». European Journal of Operational Research 85, pp. 282-296.

KATAOCA, S. (1963). «A Stochastic Programming Model». Econometrica, 31, pp. 181-196

MARKOWITZ,H. (1952). «Portfolio Selection», Journal of Finance, 7,77-91. (Se puede consultar la versión en castellano: «Selección de carteras», Cuadernos del ICE, Enero de 1991, pp. I1-19.

MARKOWITZ, H. ( 1959). «Portfolio Selection: Efficient diversification of investment». Yale University Press.

MARKOWITZ, H. y PEROLD, F. (1981): «Portfolio Analysis with Factors and Scenarios». The Journal of Finance, Vol. $36 \mathrm{~N}^{\circ} \mid 4$.
MICHAUD, R. (1989). «The Markowitz Optimization Enigma: Is «Optimized» Optimal?». Financial Analyst Journal. January-February, pp. 3 I-42.

MULVEY, J.M., VANDERBEI, R.J. AND ZENIOS, S.A. ( 1 995). «Robust optimization of large scale systems».Operations Research 43, pp. 264-28I.

ROCKAFELLAR AND R. T.WETS. (1993). «Scenario and policy aggregation in optimization under uncertainty). Mathematics of Operations Research, Vol 16: |19-147.

ROY,A. D. ( 1952). «Safety First and the Holding of Assets», Econometrica, 20, pp. 431-448. 